Brit. J. soc. Med. (1950), 4, 137-142.

\title{
THE CARE OF THE CHRONIC SICK
}

\section{A COMPARISON OF DATA FROM HOSPITALS IN THE STOKE-ON-TRENT AREA WITH DATA FROM WESTERN ROAD INFIRMARY, BIRMINGHAM*}

\author{
BY \\ C. R. LOWE and THOMAS MCKEOWN \\ From the Department of Social Medicine, University of Birmingham
}

We have previously described the results of an investigation of the chronic sick in one large hospital in the City of Birmingham (Lowe and McKeown, 1949, 1950). Before accepting conclusions based on this inquiry as representative of other parts of the country, it seemed desirable to record data from other hospitals for comparison.

\section{SUBJECT OF INQUIRY}

We selected four hospitals which draw their patients from urban and rural districts in the Stoke-on-Trent area, and which differ in size and character from the Birmingham hospital. Brief particulars of these institutions are as follows:

(i) City General Hospital, Newcastle-under-Lyme (formerly the London Road Institution). A general hospital with 915 beds distributed thus:

$\begin{array}{ll}\text { Beds for the acute sick } & 411 \\ \text { Psychiatric beds } & 179 \\ \text { Other special beds } & 112 \\ \text { Chronic sick beds } & 213\end{array}$

The chronic sick wards are arranged as part of the general hospital.

(ii) Turnhurst Road Institution, Stoke-on-Trent.-An old Public Assistance institution, with 212 beds for the chronic sick, and an annexe now offering accommodation under the provisions of Part III of the National Assistance Act (1948). No general hospital facilities are available, and patients in need of attention are transferred to the City General Hospital.

(iii) Moorlands Infirmary, Leek.-An institution of the same type as (ii), with 81 beds for the chronic sick.

(iv) Cheadle Infirmary.-A third institution of the same type as (ii), with 67 beds for the chronic sick.

\section{RESULTS OF INQUIRY}

The inquiry was identical with the earlier one at Western Road Infirmary, and was conducted by two doctors and one social worker, who spent a preliminary period on the Birmingham survey to ensure uniform records. The analysis was completed as before, and the results of most interest are here compared with those already communicated.

* This research was assisted by a grant from the Birmingham University Students' Social Services Fund. Parts I and II were published in this Journal in July 1949 and April 1950. Part III appeared in the British Medical Journal (1950) 1, 323. 
The Hospital Population.-Unfortunately we cannot, as in Birmingham, relate the hospital patients to the general population from which they are drawn, and cannot therefore give age specific rates. The results for Stoke, compared with those for Western Road Infirmary, are these:

(a) the patients are a little younger (Table I);

(b) more patients have been in hospital less than one year (Table II);

(c) the proportion of unmarried patients is higher (Table III).

TABLE I*

Percentage Age and Sex Distribution of Patients

\begin{tabular}{|c|c|c|c|c|c|c|c|c|c|c|}
\hline \multicolumn{2}{|c|}{ Age Groups (years) } & $0-29$ & $30-39$ & $40-49$ & $50-59$ & $60-69$ & 70-79 & $80+$ & Unknown & Totals \\
\hline \multirow{3}{*}{ Stoke } & Males & $\begin{array}{l}0.9 \\
\text { (1) }\end{array}$ & $\begin{array}{l}2 \cdot 2 \\
(6)\end{array}$ & $\begin{array}{l}4 \cdot 0 \\
(9)\end{array}$ & $\begin{array}{l}8 \cdot 8 \\
(20)\end{array}$ & $\begin{array}{l}18 \cdot 6 \\
(42)\end{array}$ & $\begin{array}{l}40 \cdot 3 \\
(91)\end{array}$ & $\begin{array}{l}22 \cdot 6 \\
(51)\end{array}$ & $\begin{array}{l}2 \cdot 6 \\
(6)\end{array}$ & $\begin{array}{l}100 \\
(226)\end{array}$ \\
\hline & Females & $\begin{array}{l}1 \cdot 4 \\
(4)\end{array}$ & $\begin{array}{l}2 \cdot 6 \\
(10)\end{array}$ & $\begin{array}{l}7 \cdot 2 \\
(25)\end{array}$ & $\begin{array}{l}8 \cdot 1 \\
(28)\end{array}$ & $\begin{array}{l}17 \cdot 3 \\
(60)\end{array}$ & $\begin{array}{l}31 \cdot 7 \\
(110)\end{array}$ & $\begin{array}{l}30 \cdot 8 \\
(107)\end{array}$ & $\begin{array}{l}0.9 \\
(3)\end{array}$ & $\begin{array}{l}100 \\
(347)\end{array}$ \\
\hline & $\begin{array}{l}\text { All } \\
\text { patients }\end{array}$ & $\begin{array}{l}1 \cdot 2 \\
(7)\end{array}$ & $\begin{array}{l}2 \cdot 4 \\
(14)\end{array}$ & $\begin{array}{l}5 \cdot 9 \\
(34)\end{array}$ & $\begin{array}{r}8 \cdot 4 \\
(48)\end{array}$ & $\begin{array}{l}17 \cdot 8 \\
(102)\end{array}$ & $\begin{array}{c}35 \cdot 1 \\
(201)\end{array}$ & $\begin{array}{l}27 \cdot 6 \\
(158)\end{array}$ & $\begin{array}{l}1 \cdot 6 \\
(9)\end{array}$ & $\begin{array}{l}100 \\
(573)\end{array}$ \\
\hline $\begin{array}{l}\text { Western Road } \\
\text { Infirmary }\end{array}$ & $\begin{array}{l}\text { All } \\
\text { patients }\end{array}$ & $\begin{array}{l}0 \cdot 3 \\
(3)\end{array}$ & $\begin{array}{l}0.6 \\
(6)\end{array}$ & $\begin{array}{l}2 \cdot 9 \\
(29)\end{array}$ & $\begin{array}{l}4 \cdot 6 \\
(46)\end{array}$ & $\begin{array}{l}18 \cdot 8 \\
(189)\end{array}$ & $\begin{array}{l}41 \cdot 5 \\
(417)\end{array}$ & $\begin{array}{l}31 \cdot 3 \\
(315)\end{array}$ & - & $\begin{array}{c}100 \\
(1,005)\end{array}$ \\
\hline
\end{tabular}

* In all tables percentages are shown first and actual numbers in brackets below.

TABLE II

Duration of Stay in Hospital

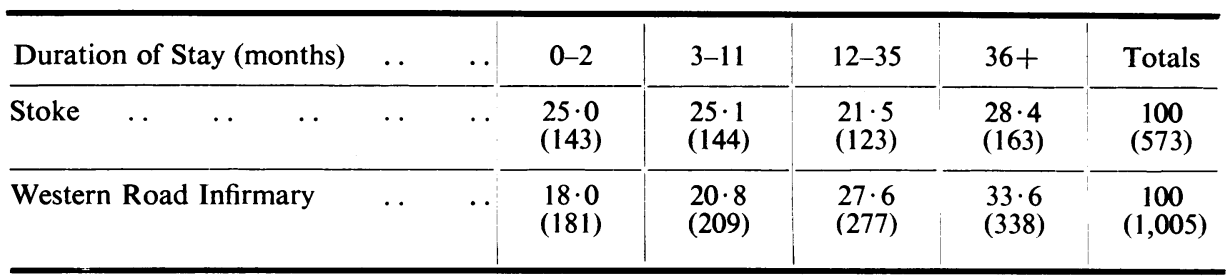

TABLE III

Marital Status of Patients

\begin{tabular}{|c|c|c|c|c|c|c|c|c|c|}
\hline \multirow{2}{*}{$\begin{array}{l}\text { Sex . } \\
\text { Status }\end{array}$} & \multirow{2}{*}{$\begin{array}{l}\cdots \\
\cdots\end{array}$} & \multicolumn{3}{|c|}{ Male } & \multirow[b]{2}{*}{ Totals } & \multicolumn{4}{|c|}{ Female } \\
\hline & & Single & Married & $\begin{array}{l}\text { Widow- } \\
\text { ers and } \\
\text { divorced }\end{array}$ & & Single & Married & $\begin{array}{l}\text { Widows } \\
\text { and } \\
\text { divorced }\end{array}$ & Totals \\
\hline Stoke & . & $\begin{array}{l}31 \cdot 4 \\
(71)\end{array}$ & $\begin{array}{l}24 \cdot 8 \\
(56)\end{array}$ & $\begin{array}{l}43 \cdot 8 \\
(99)\end{array}$ & $\begin{array}{l}100 \\
(226)\end{array}$ & $\begin{array}{l}31 \cdot 7 \\
(110)\end{array}$ & $\begin{array}{l}16 \cdot 1 \\
(56)\end{array}$ & $\begin{array}{l}52 \cdot 2 \\
(181)\end{array}$ & $\begin{array}{c}100 \\
(347)\end{array}$ \\
\hline \multicolumn{2}{|c|}{$\begin{array}{l}\text { Western Road } \\
\text { Infirmary }\end{array}$} & $\begin{array}{l}21 \cdot 1 \\
(96)\end{array}$ & $\begin{array}{l}29 \cdot 2 \\
(133)\end{array}$ & $\begin{array}{l}49 \cdot 7 \\
(226)\end{array}$ & $\begin{array}{c}100 \\
(455)\end{array}$ & $\begin{array}{l}19 \cdot 6 \\
(108)\end{array}$ & $\begin{array}{l}12 \cdot 6 \\
(69)\end{array}$ & $\begin{array}{l}67 \cdot 8 \\
(373)\end{array}$ & $\begin{array}{c}100 \\
(550)\end{array}$ \\
\hline
\end{tabular}


MEDICAL AND NURSING ReQUiREMENTS, AND DisPosAl.-As previously, patients' requirements were assessed in respect of medical attention, skilled nursing, and simple nursing. The proportion needing frequent medical attention or skilled nursing is slightly higher at Stoke (Table IV); this observation, which is true for each of the four hospitals (Table V), is not explained by the inclusion of the general hospital (City General at Newcastle-under-Lyme). The proportion of patients needing simple nursing is 93 per cent., the same as at the Birmingham hospital.

TABLE IV

Medical Attention and Skilled Nursing Required

\begin{tabular}{|c|c|c|c|c|c|c|c|c|}
\hline \multicolumn{2}{|c|}{ Frequency of Attention } & \multirow{2}{*}{$\begin{array}{c}\text { Daily } \\
2 \cdot 8 \\
(16)\end{array}$} & \multirow{2}{*}{$\begin{array}{c}\begin{array}{c}\text { More } \\
\text { than } \\
\text { once a } \\
\text { week }\end{array} \\
5 \cdot 4 \\
(31)\end{array}$} & \multirow{2}{*}{$\begin{array}{c}\text { Weekly } \\
\begin{array}{c}16 \cdot 7 \\
(96)\end{array}\end{array}$} & \multirow{2}{*}{$\begin{array}{c}\begin{array}{c}\text { Fort- } \\
\text { nightly }\end{array} \\
9 \cdot 3 \\
(53)\end{array}$} & \multirow{2}{*}{$\begin{array}{c}\begin{array}{c}\text { Monthly } \\
\text { or less }\end{array} \\
\begin{array}{l}65 \cdot 8 \\
(377)\end{array}\end{array}$} & \multirow{2}{*}{$\begin{array}{c}\text { None } \\
-\end{array}$} & \multirow{2}{*}{$\begin{array}{c}\text { Totals } \\
\begin{array}{c}100 \\
(573)\end{array}\end{array}$} \\
\hline \multirow{2}{*}{$\begin{array}{l}\text { Medical } \\
\quad \text { attention }\end{array}$} & Stoke .. & & & & & & & \\
\hline & $\begin{array}{l}\text { Western Road } \\
\text { Infirmary }\end{array}$ & $\begin{array}{l}1 \cdot 6 \\
(16)\end{array}$ & $\begin{array}{l}5 \cdot 8 \\
(58)\end{array}$ & $\begin{array}{l}10 \cdot 2 \\
(103)\end{array}$ & $\begin{array}{l}8 \cdot 5 \\
(85)\end{array}$ & $\begin{array}{l}70 \cdot 9 \\
(713)\end{array}$ & $\begin{array}{r}3 \cdot 0 \\
(30)\end{array}$ & $\begin{array}{c}100 \\
(1,005)\end{array}$ \\
\hline \multirow{2}{*}{$\begin{array}{l}\text { Skilled } \\
\text { nursing }\end{array}$} & Stoke .. & $\begin{array}{l}21 \cdot 5 \\
(123)\end{array}$ & $\begin{array}{l}1 \cdot 9 \\
(11)\end{array}$ & $\begin{array}{l}1 \cdot 0 \\
(6)\end{array}$ & - & - & $\begin{array}{l}75 \cdot 6 \\
(433)\end{array}$ & $\begin{array}{c}100 \\
(573)\end{array}$ \\
\hline & $\begin{array}{l}\text { Western Road } \\
\text { Infirmary }\end{array}$ & $\begin{array}{l}11 \cdot 9 \\
(120)\end{array}$ & $\begin{array}{l}3 \cdot 8 \\
(38)\end{array}$ & $\begin{array}{l}0 \cdot 3 \\
(3)\end{array}$ & $\begin{array}{l}0 \cdot 3 \\
(3)\end{array}$ & - & $\begin{array}{l}83 \cdot 7 \\
(841)\end{array}$ & $\begin{array}{c}100 \\
(1,005)\end{array}$ \\
\hline
\end{tabular}

TABLE V

SKILled NURSING ReQUiRed

\begin{tabular}{|c|c|c|c|}
\hline Institution & Required & Not Required & Total \\
\hline City General Hospital & $\begin{array}{l}27 \cdot 2 \\
(58)\end{array}$ & $\begin{array}{l}72 \cdot 8 \\
(155)\end{array}$ & $\begin{array}{c}100 \\
(213)\end{array}$ \\
\hline Turnhurst Road Institution & $\begin{array}{l}21 \cdot 6 \\
(45)\end{array}$ & $\begin{array}{l}78 \cdot 4 \\
(167)\end{array}$ & $\begin{array}{l}100 \\
(212)\end{array}$ \\
\hline Moorlands Infirmary & $\begin{array}{l}25 \cdot 9 \\
(21)\end{array}$ & $\begin{array}{l}74 \cdot 1 \\
(60)\end{array}$ & $\begin{array}{l}100 \\
(81)\end{array}$ \\
\hline Cheadle Infirmary .. & $\begin{array}{l}23 \cdot 9 \\
(16)\end{array}$ & $\begin{array}{l}76 \cdot 1 \\
(51)\end{array}$ & $\begin{array}{l}100 \\
(67)\end{array}$ \\
\hline Total in Stoke area & $\begin{array}{l}24 \cdot 4 \\
(140)\end{array}$ & $\begin{array}{l}75 \cdot 5 \\
(433)\end{array}$ & $\begin{array}{c}100 \\
(573)\end{array}$ \\
\hline Western Road Infirmary & $\begin{array}{l}16 \cdot 3 \\
(164)\end{array}$ & $\begin{array}{l}83 \cdot 7 \\
(841)\end{array}$ & $\begin{array}{c}100 \\
(1,005)\end{array}$ \\
\hline
\end{tabular}


Table VI gives the suggested disposal of the Stoke hospital patients, the criteria used being the same as previously described. About one-third are considered to require the facilities of a general hospital; about one-half might be accommodated in their own homes or in an institution other than a hospital; the remaining one-sixth need supervision because of their mental state. These proportions are not much different from those obtained at Western Road.

TABLE VI

Suggested Disposal of Patients

\begin{tabular}{|c|c|c|c|c|c|}
\hline \multirow{3}{*}{$\begin{array}{l}\text { Mental State } \\
\text { Suggested Disposal }\end{array}$} & \multicolumn{3}{|c|}{ Patients without Gross Mental Change } & \multirow{3}{*}{$\begin{array}{c}\begin{array}{c}\text { Patients with } \\
\text { Gross Mental } \\
\text { Change }\end{array} \\
\text { Hospital only }\end{array}$} & \multirow{3}{*}{ Total } \\
\hline & \multirow[b]{2}{*}{ Hospital } & \multicolumn{2}{|c|}{ Institution or own home } & & \\
\hline & & $\begin{array}{l}\text { With simple } \\
\text { nursing }\end{array}$ & $\begin{array}{l}\text { Without } \\
\text { simple } \\
\text { nursing }\end{array}$ & & \\
\hline Stoke & $\begin{array}{l}31 \cdot 2 \\
(179)\end{array}$ & $\begin{array}{l}46 \cdot 3 \\
(265)\end{array}$ & $\begin{array}{r}6 \cdot 6 \\
(38)\end{array}$ & $\begin{array}{l}15 \cdot 9 \\
(91)\end{array}$ & $\begin{array}{c}100 \\
(573)\end{array}$ \\
\hline Western Road Infirmary . . & $\begin{array}{l}18 \cdot 8 \\
(189)\end{array}$ & $\begin{array}{l}54 \cdot 6 \\
(549)\end{array}$ & $\begin{array}{r}7 \cdot 0 \\
(70)\end{array}$ & $\begin{array}{l}19 \cdot 6 \\
(197)\end{array}$ & $\begin{array}{c}100 \\
(1,005)\end{array}$ \\
\hline
\end{tabular}

TABLE VII

Domestic Resources Available to Patients

\begin{tabular}{|c|c|c|c|c|c|c|}
\hline \multirow[b]{2}{*}{ Type of Care Available } & \multirow[b]{2}{*}{. $\begin{array}{c}\text { No Home } \\
\text { Available }\end{array}$} & - & \multicolumn{2}{|c|}{ ome Available } & \multirow[b]{2}{*}{ Unknown } & \multirow[b]{2}{*}{ Total } \\
\hline & & $\begin{array}{l}\text { Without } \\
\text { facilities }\end{array}$ & $\begin{array}{l}\text { With } \\
\text { domestic } \\
\text { service } \\
\text { only }\end{array}$ & \begin{tabular}{|c} 
With \\
domestic \\
service and \\
simple \\
nursing
\end{tabular} & & \\
\hline . $\quad \cdots$ & $\begin{array}{l}57 \cdot 4 \\
(329)\end{array}$ & $\begin{array}{l}23 \cdot 1 \\
(132)\end{array}$ & $\begin{array}{l}14 \cdot 1 \\
(81)\end{array}$ & $\begin{array}{l}5 \cdot 4 \\
(31)\end{array}$ & - & $\begin{array}{c}100 \\
(573)\end{array}$ \\
\hline Western Road Infirmary . & $\begin{array}{l}55 \cdot 1 \\
(554)\end{array}$ & $\begin{array}{l}22 \cdot 8 \\
(229)\end{array}$ & $\begin{array}{l}17 \cdot 7 \\
(178)\end{array}$ & $\begin{array}{l}1 \cdot 8 \\
(18)\end{array}$ & $\begin{array}{l}2 \cdot 6 \\
(26)\end{array}$ & $\begin{array}{c}100 \\
(1,005)\end{array}$ \\
\hline
\end{tabular}

TABLE VIII

Source of Patients (I.E. Whence admitted)

\begin{tabular}{|c|c|c|c|c|c|c|}
\hline \multirow[t]{2}{*}{ Whence Admitted } & \multicolumn{2}{|c|}{ General Hospital } & \multirow{2}{*}{$\begin{array}{c}\text { Other } \\
\text { Hospitals } \\
\text { or Nursing } \\
\text { Homes }\end{array}$} & \multirow{2}{*}{$\begin{array}{l}\text { Direct } \\
\text { from } \\
\text { Home }\end{array}$} & \multirow[t]{2}{*}{ Unknown } & \multirow[t]{2}{*}{ Total } \\
\hline & In-patient & Out-patient & & & & \\
\hline Stoke & $\begin{array}{l}6 \cdot 5 \\
(37)\end{array}$ & $\begin{array}{l}0.5 \\
(3)\end{array}$ & $\begin{array}{l}5 \cdot 6 \\
(32)\end{array}$ & $\begin{array}{l}86 \cdot 9 \\
(498)\end{array}$ & $\begin{array}{l}0 \cdot 5 \\
(3)\end{array}$ & $\begin{array}{l}100 \\
(573)\end{array}$ \\
\hline Western Road Infirmary & $\begin{array}{l}14 \cdot 0 \\
(141)\end{array}$ & $\begin{array}{c}5 \cdot 1 \\
(51)\end{array}$ & $\begin{array}{l}3 \cdot 0 \\
(30)\end{array}$ & $\begin{array}{l}75 \cdot 7 \\
(761)\end{array}$ & $\begin{array}{l}2 \cdot 2 \\
(22)\end{array}$ & $\begin{array}{c}100 \\
(1,005)\end{array}$ \\
\hline
\end{tabular}


Table VII shows the domestic resources of patients in hospital, and once again gives no grounds for optimism about disposal to their own homes of patients now in hospital.

Adequacy of Medical Services Received.-We attempted to assess the adequacy of services received by patients at Western Road Infirmary, and used this information as a crude index of the reduction of the dimensions of the problem which might be expected to result from fuller services (McKeown and Lowe, 1950). The features examined were these:

(a) Source of patients (Table VIII). The proportion admitted through a general hospital is lower at Stoke; 87 per cent. entered directly from their homes. As

TABLE IX

INCIDENCE OF INCONTINENCE

\begin{tabular}{|c|c|c|c|c|c|c|c|}
\hline \multirow{2}{*}{ Continence. . } & \multirow{2}{*}{$\ldots$} & \multirow{2}{*}{$\cdots$} & \multirow{2}{*}{ Continent } & \multicolumn{2}{|r|}{ Incontinent } & & \multirow{2}{*}{ Total } \\
\hline & & & & Urine only & Faeces only & $\begin{array}{l}\text { Urine and } \\
\text { faeces }\end{array}$ & \\
\hline Stoke & $\cdots$ & $\cdots$ & $\begin{array}{l}64 \cdot 9 \\
(368)\end{array}$ & $\begin{array}{l}5 \cdot 8 \\
(33)\end{array}$ & $\begin{array}{c}1 \cdot 6 \\
(9)\end{array}$ & $\begin{array}{l}27 \cdot 7 \\
(157)\end{array}$ & $\begin{array}{l}100 \\
(567)^{*}\end{array}$ \\
\hline \multicolumn{3}{|c|}{ Western Road Infirmary .. } & $\begin{array}{l}65 \cdot 2 \\
(648)\end{array}$ & $\begin{array}{l}7 \cdot 6 \\
(75)\end{array}$ & $\begin{array}{l}1 \cdot 2 \\
(12)\end{array}$ & $\begin{array}{l}26 \cdot 0 \\
(258)\end{array}$ & $\begin{array}{l}100 \\
(993) \dagger\end{array}$ \\
\hline
\end{tabular}

* Six patients excluded: two, colostomy for carcinoma; four, suprapubic cystotomy.

$\dagger$ Twelve patients excluded: five, colostomy for carcinoma; seven, suprapubic cystotomy.

TABLE $X$

Mobility of PATIENTS

\begin{tabular}{|c|c|c|c|c|c|c|c|c|c|}
\hline \multirow{2}{*}{ Mobility } & \multirow{2}{*}{.. } & \multicolumn{2}{|c|}{ Bedfast } & \multicolumn{2}{|c|}{ Sitting out of Bed } & \multicolumn{3}{|c|}{ Ambulant } & \multirow{2}{*}{ Total } \\
\hline & & Lying & Sitting & $\begin{array}{l}\text { With } \\
\text { help }\end{array}$ & $\begin{array}{l}\text { Without } \\
\text { help }\end{array}$ & No.stairs & Stairs & $\begin{array}{l}\text { Out of } \\
\text { doors }\end{array}$ & \\
\hline Stoke & . & $\begin{array}{l}25 \cdot 0 \\
(143)\end{array}$ & $\begin{array}{l}29 \cdot 2 \\
(167)\end{array}$ & $\begin{array}{l}12 \cdot 0 \\
(69)\end{array}$ & $\begin{array}{l}7 \cdot 3 \\
(42)\end{array}$ & $\begin{array}{l}10 \cdot 5 \\
(60)\end{array}$ & $\begin{array}{l}3 \cdot 3 \\
(19)\end{array}$ & $\begin{array}{l}12 \cdot 7 \\
(73)\end{array}$ & $\begin{array}{c}100 \\
(573)\end{array}$ \\
\hline \multicolumn{2}{|c|}{$\begin{array}{l}\text { Western Road } \\
\text { Infirmary }\end{array}$} & $\begin{array}{l}28 \cdot 8 \\
(289)\end{array}$ & $\begin{array}{l}29 \cdot 7 \\
(299)\end{array}$ & $\begin{array}{l}12 \cdot 2 \\
(123)\end{array}$ & $\begin{array}{l}4 \cdot 8 \\
(48)\end{array}$ & $\begin{array}{l}15 \cdot 4 \\
(155)\end{array}$ & $\begin{array}{l}2 \cdot 1 \\
(21)\end{array}$ & $\begin{array}{l}7 \cdot 0 \\
(70)\end{array}$ & $\begin{array}{c}100 \\
(1,005)\end{array}$ \\
\hline
\end{tabular}

TABLE XI

Mental State of Patients

\begin{tabular}{|c|c|c|c|c|c|c|c|}
\hline \multirow{2}{*}{ Mental State } & \multirow{2}{*}{$\cdots$} & \multirow{2}{*}{$\ldots$} & \multirow{2}{*}{ Normal } & \multicolumn{2}{|c|}{ Abnormal } & \multirow{2}{*}{ Unknown } & \multirow{2}{*}{ Total } \\
\hline & & & & $\begin{array}{c}\text { Need } \\
\text { supervision }\end{array}$ & $\begin{array}{l}\text { Need no } \\
\text { supervision }\end{array}$ & & \\
\hline Stoke & $\ldots$ & $\ldots$ & $\begin{array}{l}49 \cdot 2 \\
(282)\end{array}$ & $\begin{array}{l}15 \cdot 9 \\
(91)\end{array}$ & $\begin{array}{l}34 \cdot 2 \\
(196)\end{array}$ & $\begin{array}{l}0.7 \\
(4)\end{array}$ & $\begin{array}{c}100 \\
(573)\end{array}$ \\
\hline \multicolumn{3}{|c|}{ Western Road Infirmary . } & $\begin{array}{l}64 \cdot 0 \\
(643)\end{array}$ & $\begin{array}{l}19 \cdot 6 \\
(197)\end{array}$ & $\begin{array}{l}14 \cdot 0 \\
(141)\end{array}$ & $\begin{array}{l}2 \cdot 4 \\
(24)\end{array}$ & $\begin{array}{c}100 \\
(1,005)\end{array}$ \\
\hline
\end{tabular}


at Western Road Infirmary, only about one-quarter of all patients are known to have had laboratory or out-patient investigation before their admission.

(b) Continence, mobility, and mental state. The relevant comparisons are recorded in Tables IX, X, and XI respectively. The proportion of incontinents and of bedfast patients is almost identical in the two surveys; the proportion needing supervision because of their abnormal mental state is a little lower at Stoke.

\section{SUMMARY}

An investigation of chronic sick in four hospitals drawing their patients from the Stoke-on-Trent area gave results which differ very little from those recorded for Western Road Infirmary, Birmingham. In particular, conclusions about disposal based on the Birmingham hospital are not disturbed by the additional data now available.

We are indebted to Dr. C. G. Lewis, Medical Superintendent of the City General Hospital, whose co-operation made it possible for us to collect material from the Stoke area, to Dr. P. J. Sangster and Dr. S. J. Wainwright, who completed the medical records, and to Miss G. E. Pountney, who collected the social data.

REFERENCES

Lowe, C. R., and McKeown, T. (1949). British Journal of Social Medicine, 3, 110. $\longrightarrow$ (1950). Ibid., 4, 61 .

McKeown, T., and Lowe, C. R. (1950). Brit. med. J., 1, 323. 